\title{
DHEA administration and exercise training improves insulin resistance in obese rats
}

\author{
Koji Sato ${ }^{1}$, Motoyuki lemitsu', Katsuji Aizawa ${ }^{2}$, Noboru Mesaki ${ }^{3}$, Ryuichi Ajisaka ${ }^{4}$ and Satoshi Fujita ${ }^{1 *}$
}

\begin{abstract}
Background: Dehydroepiandrosterone (DHEA) is precursor of sex steroid hormone. We demonstrated that acute DHEA injection to type 1 diabetes model rats induced improvement of hyperglycemia. However, the effect of the combination of DHEA administration and exercise training on insulin resistance is still unclear. This study was undertaken to determine whether 6-weeks of DHEA administration and/or exercise training improve insulin resistance in obese male rats.

Methods: After 14 weeks of a high-sucrose diet, obese male Wistar rats were assigned randomly to one of four groups: control, DHEA administration, exercise training, and a combination of DHEA administration and exercise training ( $n=10$ each group).

Results: After 6-weeks of DHEA administration and/or exercise training, rats in the combination group weighed significantly less and had lower serum insulin levels than rats in the other groups. Moreover, the rats treated with DHEA alone or DHEA and exercise had significantly lower fasting glucose levels (combination, $84 \pm 6.5 \mathrm{mg} / \mathrm{dL}$; DHEA, $102 \pm 9.5 \mathrm{mg} / \mathrm{dL}$; control, $148 \pm 10.5 \mathrm{mg} / \mathrm{dL}$ ). In addition, insulin sensitivity check index showed significant improvements in the combination group (combination, $0.347 \pm 0.11$; exercise, $0.337 \pm 0.16 \%$; DHEA, $0.331 \pm 0.14$; control, $0.308 \pm 0.12$ ). Muscular DHEA and $5 a$-dihydrotestosterone (DHT) concentrations were significantly higher in the combination group, and closely correlated with the quantitative insulin-sensitivity check index (DHEA: $r=0.71$, $p<0.01 ; \mathrm{DHT}: r=0.69, p<0.01)$.
\end{abstract}

Conclusion: These results showed that a combination of DHEA administration and exercise training effectively improved fasting blood glucose and insulin levels, and insulin sensitivity, which may reflect increased muscular DHEA and DHT concentrations.

Keywords: Exercise training, Insulin sensitivity, Sex steroid hormone, Obesity

\section{Introduction}

Obesity is one of the risk factors for type 2 diabetes, cardiovascular diseases, hypertension, and dyslipidemia. The obesity and type 2 diabetes patients show lower concentrations of dehydroepiandrosterone (DHEA) and other sex steroid hormone [1-3]. Decreased DHEA levels in obese patients or those with type 2 diabetes are related to insulininduced inhibition of enzyme activity for adrenal androgen synthesis [4]. However, DHEA administration and exercise leads to increases in muscular steroidogenesis-related enzymes and concomitant increases in plasma and muscular DHEA and $5 \alpha$-dihydrotestosterone (DHT) levels in rats

\footnotetext{
* Correspondence: safujita@fc.ritsumei.ac.jp

'Faculty of Sport and Health Science, Ritsumeikan University, Kusatsu, Shiga, Japan

Full list of author information is available at the end of the article
}

[5-7]. Moreover, several reports demonstrated that shortterm (2 weeks) DHEA administration induced an acute decrease in blood glucose levels in mice [8,9], reduced serum insulin levels in older rats $[2,10,11]$, and enhanced the activities of enzyme related to hepatic glucose metabolismin diet-induced or Zucker obese rats [12,13]. Recently, we have reported that acute DHEA injections improved hyperglycemia in streptozotocin (STZ)-induced diabetic rats [7]. In addition, Han and colleagues [14] demonstrated an enhanced insulin sensitivity in response to 2 weeks of wheel-running exercise and DHEA administration in 25-month-old rats, although additive effects of the treatments were not apparent.

DHEA and its sulfate derivate (DHEA-S) are precursors of sex steroid hormones, that circulate in blood before

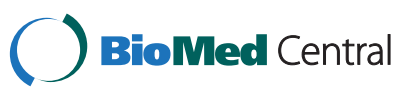


they are used by target tissues. Generally, DHEA is produced by adrenal, but several studies demonstrated that brain, liver, bone and any other tissues can also produce DHEA in both human and rodents [15-17]. Recently, we demonstrated that skeletal muscle can locally synthesize DHT from DHEA and testosterone [18]. According to our previous studies, acute aerobic exercise enhances the local bioactive androgen metabolism, and increased DHEA and DHT levels in skeletal muscle $(5,6)$. It is still unclear whether the combination of chronic DHEA administration and aerobic exercise training increases muscular level of DHEA and DHT, and it induces the improvement of insulin resistance more effectively in obesity.

We hypothesized that, compared with either DHEA administration or exercise training treatment alone, 6-weeks of DHEA administration combined with exercise training in obese rats would produce larger effects on steroidogenesis from DHEA to DHT in skeletal muscle and improvements in insulin sensitivity. To test this hypothesis, we investigated fasting blood glucose, insulin levels, the quantitative insulin-sensitivity check index (QUICKI) as an index of insulin sensitivity after 6-weeks of DHEA administration, exercise training, or both in rats with dietinduced obesity.

\section{Methods}

Ethical approval for this study was obtained from the Committee on Animal Care at the University of Tsukuba. Male Wistar rats (220-250 g, 10 weeks old; Charles River Japan, Kanagawa, Japan) were cared for according to The Guiding Principles for the Care and Use of Animals based on the Declaration of Helsinki. The Wistar rats were housed individually in an animal facility under controlled conditions (12:12-h light-dark cycle). The rats were allowed water ad libitum and placed on a purified highsucrose diet ( $68 \%$ of $\mathrm{kcal}$ from sucrose, $20 \%$ from protein, and $12 \%$ from fat) for 14 weeks, according to a previous study with minor modifications [19]. After 14 weeks of the high-sucrose diet, the animals were randomly assigned to one of four groups: sedentary rats administered sesame oil (control group, $n=10$ ), obese rats treated with DHEA (DHEA group, $n=10$ ), obese rats subjected to exercise training (exercise group, $n=10$ ), and obese rats treated with DHEA and subjected to exercise training (combination group, $n=10$ ). DHEA was obtained from Wako Pure Chemical Industries (Osaka, Japan). All animals continued on the high-sucrose diet during the 6-week experimental period. In the DHEA and combination groups, DHEA ( $1 \mathrm{mg} / \mathrm{kg}$ body weight) dissolved in sesame oil was administered orally every day for 6 weeks. In the control group, the same amount of vehicle (sesame oil) was administered orally every day. Body weight and dietary intake were measured every week during the experiment. Posttraining experiments in trained rats were performed $48 \mathrm{~h}$ after the last round of exercise training. After all other measurements were obtained the soleus and gastrocnemius muscles were quickly removed, weighed, rinsed in ice-cold saline, and frozen in liquid nitrogen.

\section{Exercise protocol}

The obese exercise training group was trained on a rodent treadmill (KN-73, Natsume Seisakusyo, Tokyo, Japan) at about $10-25 \mathrm{~m} / \mathrm{min}$ over a period of 3 days for accustomed to the treadmill. The rats then ran on the treadmill for $1 \mathrm{~h}$ at $25 \mathrm{~m} / \mathrm{min}$ without incline 5 days/week for 6 weeks. The intensity, duration and time of the exercise was kept constant during the rest of the training period.

\section{Protein assay and muscle concentrations of DHEA and DHT}

Muscle specimens were homogenized in $20 \mathrm{mM}$ Tris- $\mathrm{HCl}$ (pH 7.8), $300 \mathrm{mM} \mathrm{NaCl}, 2 \mathrm{mM}$ ethylenediaminetetraacetic acid (EDTA), $2 \mathrm{mM}$ dithiothreitol (DTT), 2\% nonident P-40, 0.2\% sodium lauryl sulphate (SDS), 0.2\% sodium deoxycholate, $0.5 \mathrm{mM}$ phenylmethylsulfonyl fluoride, $60 \mu \mathrm{g} / \mathrm{ml}$ aprotinin, and $1 \mu \mathrm{g} / \mathrm{ml}$ leupeptin. Homogenates were rotated slowly for $30 \mathrm{~min}$ at $4^{\circ} \mathrm{C}$ and then centrifuged at $12,000 \times g$ for $15 \mathrm{~min}$ at $4^{\circ} \mathrm{C}$. The protein concentration of the resulting supernatant was determined. For the determination of DHEA and DHT levels, muscle samples were diluted by 200 times with each assay buffer. The levels of DHEA and DHT in skeletal muscle extracts were determined using Enzyme-Linked Immuno Sorbent Assay (ELISA) kit (Assay Designs, Ann Arbor, MI, IBL Hamburg, Germany). The techniques and materials used in these analyses followed the manufacturer's protocol. The immobilized polyclonal antibodies were raised against DHEA and DHT, whereas the secondary horseradishperoxidase-coupled antibodies were monoclonal. Optical density at $450 \mathrm{~nm}$ was measured on a microplate reader (BioLumin 960; Molecular Dynamics, Tokyo, Japan). All samples were assayed in duplicate.

\section{Glucose and insulin concentrations}

Serum insulin concentrations were measured using an ELISA kit (Shibayagi Co, Gunma, Japan), according to the manufacturer's protocol. All samples were assayed in duplicate. Optical density at $450 \mathrm{~nm}$ was qualified using a microplate reader (BioLumin 960; Molecular Dynamics, Tokyo, Japan). Fasting glucose was assessed from the tail vein before and after the treatment period under overnight fasting condition. Glucose concentrations were assessed three times from the tail vein using a blood glucose meter (Ascensia, Bayer HealthCare, Tokyo, Japan).

\section{Insulin sensitivity}

QUICKI was calculated according to the previous study from fasting glucose and insulin vaues [20-22]. QUICKI = 
$1 /\left[\log \left(\mathrm{I}_{0}\right)+\log \left(\mathrm{G}_{0}\right)\right]$, where $\mathrm{I}_{0}$ is fasting insulin $(\mu \mathrm{U} / \mathrm{ml})$, and $\mathrm{G}_{0}$ is fasting glucose $(\mathrm{mg} / \mathrm{dl})$.

\section{Statistical analysis}

All values are expressed as means \pm SE. Statistical evaluations were performed using repeated measures twoway ANOVA (time $\times$ group) for weight changes. A post-hoc comparison test was used to correct for multiple comparisons (Bonferroni test) when analyses revealed significant differences. For ANOVA, $P<0.01$ was considered significant. Relationships between sex steroid hormone concentrations and the quantitative insulin-sensitivity check index (QUICKI) was determined using Pearson correlation coefficients.

\section{Results}

\section{Body weight}

Body weight before the treatment periods did not differ among the groups. After 6 weeks of DHEA administration and/or exercise training, body weight was significantly $(p<0.01)$ lower in the combination group than in the control group. After 5 weeks, body weight was significantly $(p<0.01)$ lower in the combination group than in the DHEA group or the exercise group (Figure 1). Final body weights in the DHEA and exercise groups were significantly lower than in the control group. Moreover, the combination group showed a significantly lower body weight than the other groups (Figure 1).

\section{Dietary intake, muscle weight and epididymal fat weight} Exercise training and DHEA administration did not decrease food intake of the rats. Average food intake during the 6 weeks was $19.74 \pm 0.62 \mathrm{~g} /$ day in the control group, $19.64 \pm 0.37 \mathrm{~g} /$ day in the DHEA group, $19.61 \pm 0.43 \mathrm{~g} /$ day in the exercise group, and $19.59 \pm 0.53 \mathrm{~g} /$ day in the combination group. No significant difference in the food intake was found among groups during the treatment period (Table 1).

Table 1 shows the weights of the soleus and gastrocnemius muscles after the treatment period. Marked increases in the weight of the soleus and gastrocnemius muscles were observed in the exercise and combination groups compared with the control and DHEA groups. The soleus and gastrocnemius muscles weighed significantly more in the DHEA group than in the control group $(p<0.01$, Table 1$)$. Epididymal fat weight was significantly decreased in combination group compared to control and exercise group $(p<0.01$, Table 1$)$.

Fasting glucose levels, serum insulin levels, and QUICKI The fasting glucose levels were not significantly different among groups before the treatment periods (Table 1). After 6 weeks of DHEA administration and/or exercise training, fasting glucose levels were significantly lower in the DHEA, exercise, and combination groups than in the control group. Moreover, fasting glucose levels in the combination group were significantly lower than in the DHEA group (Table 1).

After 6 weeks of DHEA administration and/or exercise training, the serum insulin levels were significantly lower in the DHEA, exercise, and combination groups than in the control group. Serum insulin levels were also significantly lower in the combination group than in the other groups (Figure 2A). Similarly, QUICKI were significantly higher in the DHEA, exercise, and the combination groups than in the control group $(p<0.01$. Figure 2B). Of particular interest, the QUICKI were significantly higher in the combination group than in the other groups $(p<0.01)$. No significant correlation between body weight and fasting glucose, or body weight and QUICKI was found in the present study.

\section{Intramuscular DHEA and DHT concentrations}

Intramuscular DHEA and DHT concentrations were significantly greater in the DHEA, exercise, and combination groups than in the control group. The DHEA and DHT concentrations were also significantly greater in the combination group than in either the DHEA or exercise group (Figures $3 \mathrm{~A}$ and $3 \mathrm{~B})$. Of note, intramuscular DHEA and DHT concentrations significantly correlated

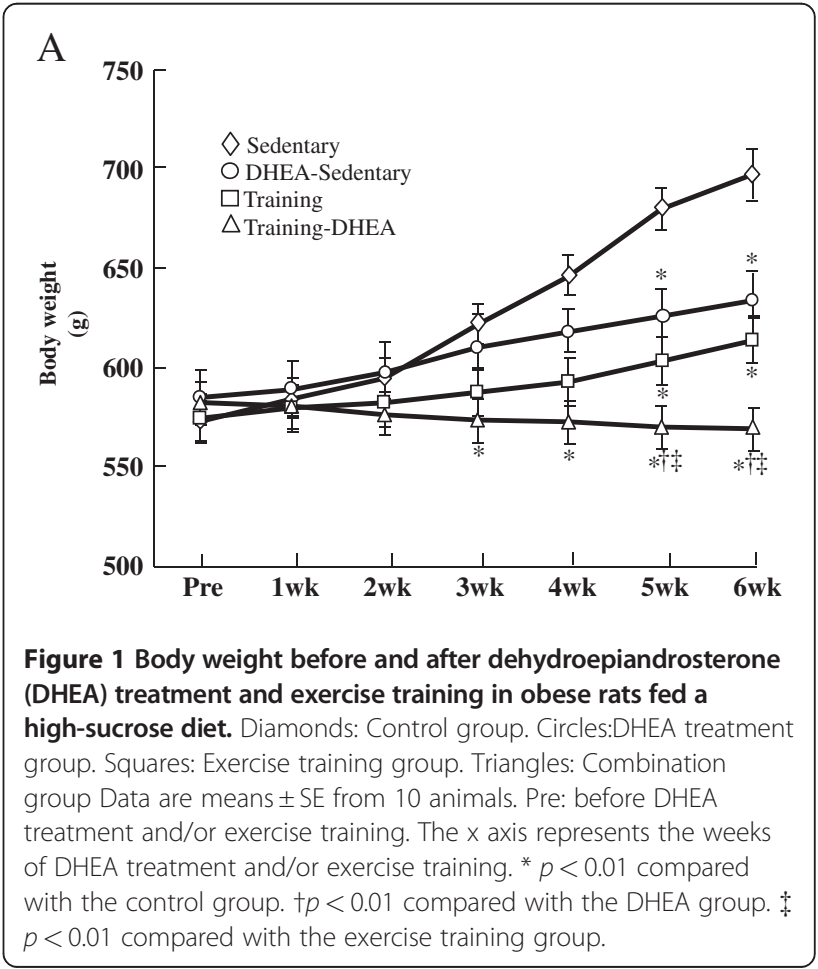


Table 1 Animal characteristics

\begin{tabular}{|c|c|c|c|c|}
\hline & Sedentary $(n=10)$ & $\operatorname{DHEA}(n=10)$ & Training $(n=10)$ & Training-DHEA $(n=10)$ \\
\hline \multicolumn{5}{|c|}{ Muscle weight (g/body weight) } \\
\hline Soleus & $0.32 \pm 0.02$ & $0.48 \pm 0.04 *$ & $0.56 \pm 0.03 *+$ & $0.57 \pm 0.04 *+$ \\
\hline Gastrocnemius & $3.63 \pm 0.6$ & $4.31 \pm 0.3 *$ & $4.66 \pm 0.3 *+$ & $4.73 \pm 0.3 *+$ \\
\hline Epididymal fat (g) & $38.2 \pm 4.8$ & $27.4 \pm 3.6 *$ & $33.8 \pm 2.8$ & $23.7 \pm 4.1 *$ \\
\hline Dietary intake(g/day) & $19.74 \pm 0.62$ & $19.64 \pm 0.37$ & $19.61 \pm 0.43$ & $19.59 \pm 0.53$ \\
\hline Pre-fasting glucose(mg/dl) & $154 \pm 11.5$ & $159 \pm 10.2$ & $162 \pm 8.7$ & $158 \pm 9.8$ \\
\hline Post-fasting glucose(mg/dl) & $148 \pm 10.5$ & $102 \pm 9.5 *$ & $92 \pm 7.8 *$ & $84 \pm 6.5 *+$ \\
\hline
\end{tabular}

DHEA:dehydroepiandrosterone.

Pre-fasting glucose: fasting glucose level before DHEA administration and/or exercise training.

Post-fasting glucose: fasting glucose level after DHEA administration and/or exercise training.

Data are means $\pm \mathrm{SE}$.

* $P<0,05$, vs Sedentary control group.

$+P<0.05$, vs DHEA treatment group.

$\$ P<0.05$, vs Training group.

with QUICKI (DHEA: $r=0.71, p<0.001$; DHT: $r=0.69$, $p<0.001$. Figure 4).

\section{Discussion}

Results from this study demonstrated that 6-weeks of DHEA treatment with exercise training induced larger decreases in fasting glucose levels than results observed for DHEA administration alone. The combined interventions were more beneficial for insulin resistance and body weight than either DHEA administration or exercise training alone. These effects in the combination group may have reflected significant increases in muscular DHEA and DHT levels, which closely correlated with QUICKI.

In the present study, both exercise training and DHEA administration significantly increased muscular concentrations of DHEA and DHT, and these increases were even more pronounced when these treatment modalities were combined. In fact, muscular DHEA and DHT concentrations significantly correlated with QUICKI in this study. Recently, a single DHEA injection in rats with STZ-induced diabetes was shown to reverse impaired GLUT-4-related signaling in muscle, such as Akt/PKC $/ \lambda$ activity and GLUT-4 translocation [7]. Additionally, shortterm exercise training is known to improve blood glucose levels and accelerate muscular glucose uptake and utilization [23]. Therefore, the combination of DHEA administration and exercise training may additively or synergistically improve blood glucose levels and activate the glucose uptake in skeletal muscle. According to Han et al. [14], plasma insulin levels decreased and the glucose infusion rate was increased by DHEA treatment and voluntary running-wheel training in 25-month-old rats. Additive effects were not observed in the combination group, however. In the present study, a 6-week regimen of DHEA administration and exercise training resulted in larger improvements in hyperglycemia and insulin resistance in the obese rats. Consequently, a longer treatment period, such as the 6-week regimen used in this study, may be required to obtain additive benefits for improvement of insulin sensitivity in diet-induced obese rats.

Although DHEA administration and exercise training each produced beneficial effects, 6-weeks of combination treatment were more effective for obesity. The precise mechanisms that reduced abdominal fat weight in the combination group remain unclear, yet we can propose several plausible hypotheses. 2 weeks of DHEA administration has been shown to activate fatty acid metabolism-related enzymes, such as long-chain fatty acyl-coenzyme A synthase, and to increase free CoA levels in liver $[12,13]$. In addition, exercise training is known to reduce adipogenesis via upregulation of fatty acid metabolism and increased energy expenditure [24]. Therefore, 6-weeks of combination treatment may have promoted additive reductions in abdominal fat volume.

Patients with metabolic syndrome have lower DHEA and DHEA-S levels [25]. We recently demonstrated that DHEA enhanced GLUT-4-relatedsignaling in cultured skeletal muscle cells [18]. Furthermore, rats with STZ-induced diabetes had lower muscle DHEA concentrations, and acute DHEA injections improved GLUT-4-relatedsignaling with increased muscular DHEA and DHT levels. Therefore, muscular DHEA and DHT levels appear to be related to blood glucose levels in obesity and type 2 diabetes. The present study showed that DHEA administration, exercise training, and combination of the two modalities significantly increased basal muscular DHEA and DHT levels. Moreover, brief exercise significantly increases muscular levels of DHEA and steroidogenesis-related enzymes $[5,6]$. Exercise training is beneficial for patients with insulin resistance and hyperinsulinemia [26,27]. Although exercise training has been shown to improve insulin resistance and glucose metabolism in obese patients with type 2 diabetes, most effects are observed after longer exercise programs. 


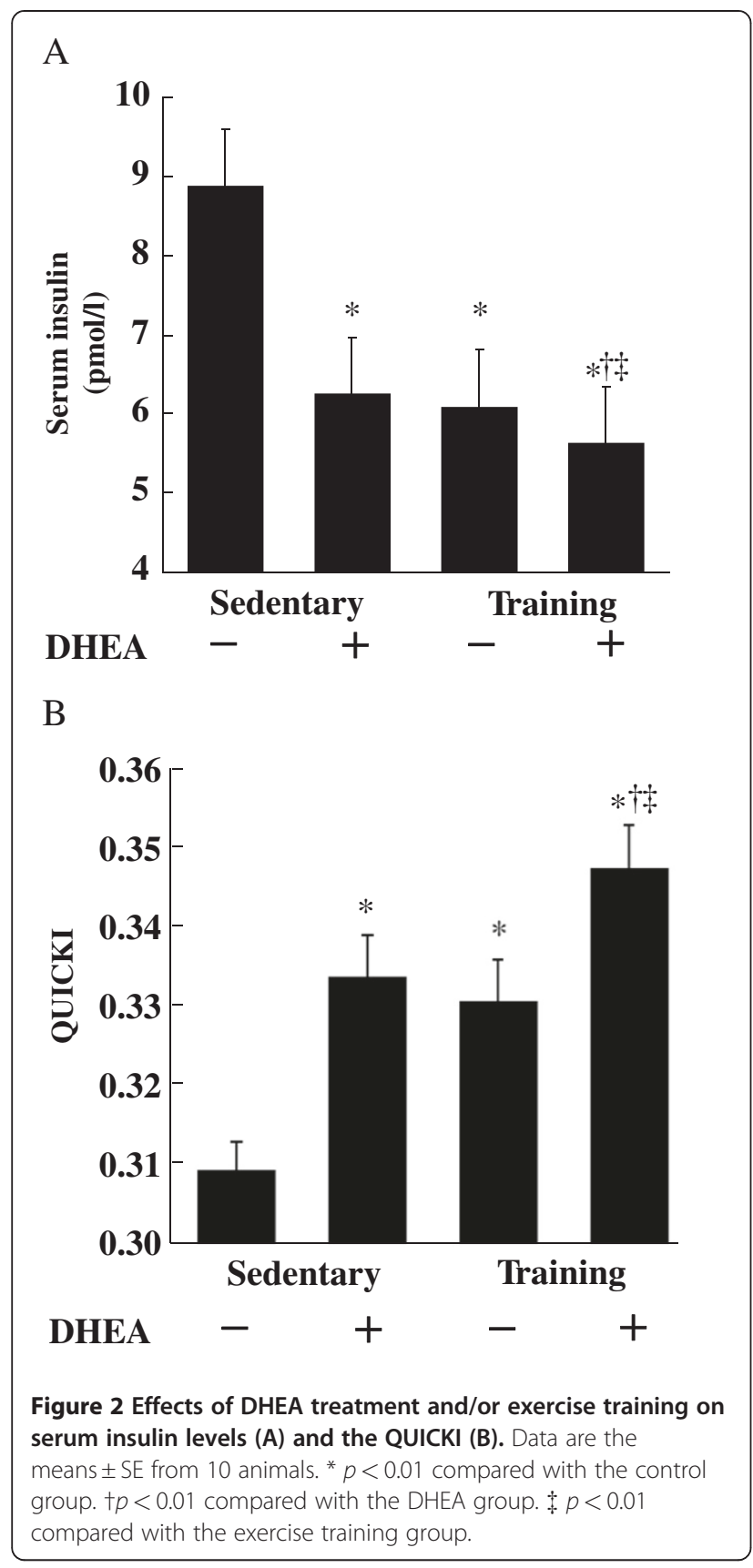

Therefore, combination treatment may be more beneficial than either therapy alone. Increasing basal muscular DHEA and DHT levels with DHEA and regular exercise may provide additive benefits for reducing abdominal fat weight and improving insulin resistance in obesity. However, the present study was conducted with relatively small number of experiments; therefore, further studies are warranted to confirm the current findings and to clarify the molecular mechanisms for combined benefits of DHEA and exercise training.

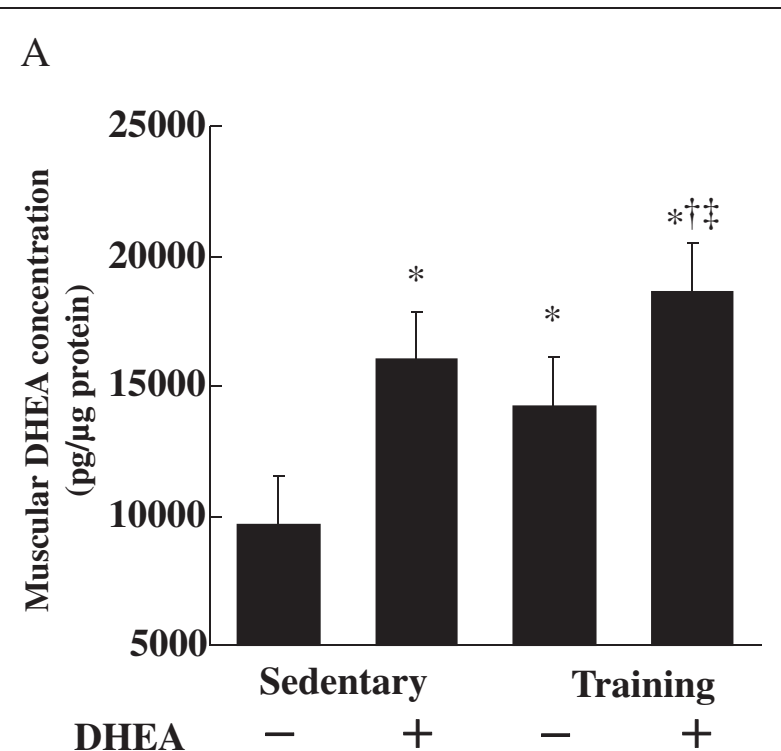

B

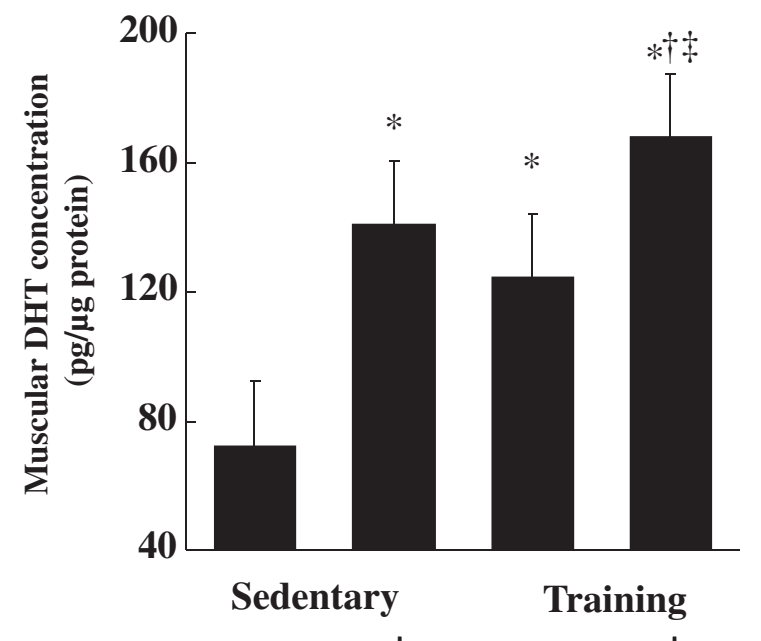

DHEA $\quad-\quad+\quad-\quad-\quad+$

Figure 3 Effects of DHEA treatment and/or exercise training on muscle DHEA and 5a-dihydrotestosterone (DHT)

concentrations. Data are means \pm SE from 10 animals. ${ }^{*} p<0.01$ compared with the control group. $\uparrow p<0.01$ compared with the DHEA group $\sharp p<0.01$ compared with the exercise training group.

Although this combined treatment modalities may provide a new therapeutic avenue for obesity and insulin resistance related to obesity, previous studies have reported some mixed results. While one study indicated that DHEA administration improved insulin sensitivity in elderly people $[28,29]$, others demonstrated no improvement in insulin sensitivity or body composition [30]. Therefore, further studies are necessary to clarify the effect of DHEA 
A

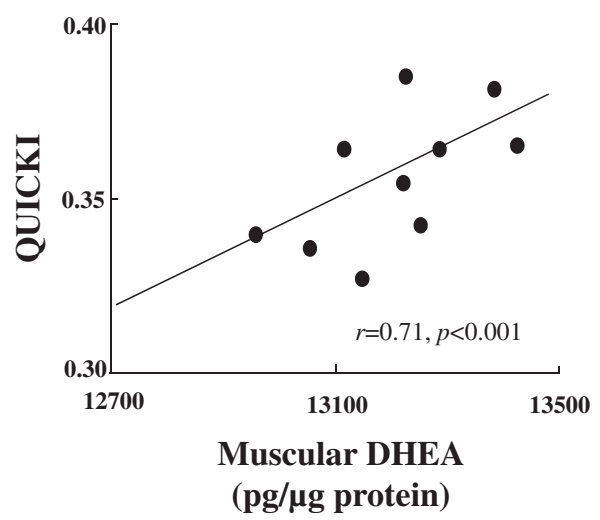

B

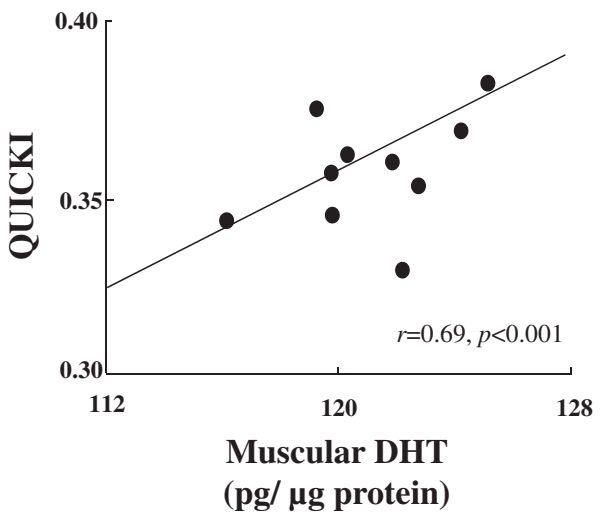

Figure 4 Correlations between muscular DHEA and the QUICKI (A) and muscular DHT and the QUICKI (B) in combination treatment groups.

supplementation not only in healthy adults but also in obese or diabetic patients.

In conclusion, the results from this study demonstrated that 6-weeks of DHEA administration combined with exercise training produced larger benefits for insulin sensitivity, body weight, and abdominal fat compared with DHEA administration or exercise training alone.

\section{Abbreviations}

DHEA: Dehydroepiandrosterone; DHEA-S: DHEA sulfate; DHT:

5a-dihydrotestosterone; QUICKI: Quantitative insulin-sensitivity check index.

\section{Competing interests}

The authors declare that they have no competing interests.

\section{Acknowledgement}

This work was supported by grants-in-aid for Scientific Research from the Ministry of Education, Culture, Sports, Science and Technology of Japan (23700849, 21300254, 22650166). This work was funded by a Sasakawa Scientific Research Grant from The Japan Science Society (24-445).

\section{Author details}

${ }^{1}$ Faculty of Sport and Health Science, Ritsumeikan University, Kusatsu, Shiga, Japan. ${ }^{2}$ Department of literature, Senshu University, Kawasaki, Kanagawa, Japan. ${ }^{3}$ University of Tsukuba, Tsukuba, Ibaraki, Japan. ${ }^{4}$ Department of Comprehensive Human Sciences, University of Tsukuba, Tsukuba, Ibaraki, Japan.

\section{Authors' contribution}

SK: AB, IM: ES, AK: ES, MN: ES, AR: FG, FS: FG. All authors read and approved the final manuscript.

Received: 28 October 2011 Accepted: 30 May 2012

Published: 30 May 2012

\section{References}

1. Berdanier CD, Parente JA Jr, McIntosh MK: Is dehydroepiandrosterone an antiobesity agent? FASEB J 1993, 7:414-419.

2. Cleary MP, Zisk JF: Anti-obesity effect of two different levels of dehydroepiandrosterone in lean and obese middle-aged female Zucker rats. Int J Obes 1986, 10:193-204.

3. Lea-Currie YR, Wen P, McIntosh MK: Dehydroepiandrosterone-sulfate (DHEAS) reduces adipocyte hyperplasia associated with feeding rats a high-fat diet. Int J ObesRelatMetabDisord 1997, 21:1058-1064.
4. Kapoor D, Malkin CJ, Channer KS, Jones TH: Androgens, insulin resistance and vascular disease in men. Clinical Endocrinology 2005, 63:239-250.

5. Aizawa K, lemitsu M, Otsuki T, Maeda S, Miyauchi T, Mesaki N: Sex differences in steroidogenesis in skeletal muscle following a single bout of exercise in rats. J Appl Physio/ 2008, 104:67-74.

6. Aizawa K, lemitsu M, Maeda S, Otsuki T, Sato K, Ushida T, Mesaki N, Akimoto T: Acute exercise activates local bioactive androgen metabolism in skeletal muscle. Steroids 2010, 75:219-223.

7. Sato K, lemitsu M, Aizawa K, Ajisaka R: DHEA improves impaired activation of Akt and PKC C/A-GLUT4 pathway in skeletal muscle and improves hyperglycaemia in streptozotocin-induced diabetes rats. Acta Physiol 2009, 197:217-225.

8. Coleman DL, Leiter EH, Schwizer RW: Therapeutic effects of dehydroepiandrosterone (DHEA) in diabetic mice. Diabetes 1982, 31:830-833.

9. Coleman DL, Schwizer RW, Leiter EH: Effect of genetic background on the therapeutic effects of dehydroepiandrosterone (DHEA) in diabetes-obesity mutants and in aged normal mice. Diabetes 1984, 33:26-32.

10. Cleary MP, Shephard A, Zisk J, Schwartz A: Effect of dehydroepiandrosterone on body weight and food intake in rats. NutrBehav 1983, 1:127-136.

11. Tagliaferro AR, Ronan AM, Payne J, Meeker LD, Tse S: Increased lipolysis to beta-adrenergic stimulation after dehydroepiandrosterone treatment in rats. Am J Physiol 1995, 268:R1374-R1380.

12. Mohan PF, Cleary MP: Effect of short-term DHEA administration on liver metabolism of lean and obese rats. Am J Physiol 1988, 255:E2-E8.

13. Mohan PF, Ihnen JS, Levin BE, Cleary MP: Effects of dehydroepiandrosterone treatment in rats with diet-induced obesity. J Nutr 1990, 120:1103-1114.

14. Han DH, Hansen PA, Chen MM: Holloszy JO.DHEA treatment reduces fat accumulation and protects against insulin resistance in male rats.J Gerontol A BiolSci. Med Sci 1998, 53:19-24.

15. Grasfeder LL, Gaillard S, Hammes SR, Ilkayeva O, Newgard CB, Hochberg RB, Dwyer MA, Chang CY, McDonnell DP: Fasting-induced hepatic production of DHEA is regulated by PGC-1[alpha], ERR[alpha], and HNF4 [alpha]. Mol Endocrinol 2009, 23:1171-1182.

16. Mellon SH, Griffin LD, Compagnone NA: Biosynthesis and action of neurosteroids. Brain Res Rev 2001, 37:3-12.

17. Takeuchi S, Mukai N, Tateishi T, Miyakawa S: Production of sex steroid hormones from DHEA in articular chondrocyte of rats. Am J Physio/ 2007, 293:E410-E415.

18. Sato K, lemitsu M, Aizawa K, Ajisaka R: Testosterone and DHEA activate the glucose metabolism-related signaling pathway in skeletal muscle. Am J Physiol 2008, 294:E961-E968.

19. Commerford SR, Ferniza JB, Bizeau ME, Thresher JS, Willis WT, Pagliassotti MJ: Diets enriched in sucrose or fat increase gluconeogenesis and G-6-Pase but not basal glucose production in rats. Am J Physiol 2002, 283:E545-E555.

20. Levy JR, Clore JN, Stevens W: Dietary $n-3$ polyunsaturated fatty acids decrease hepatic triglycerides in Fischer 344 rats. Hepatology 2004, 39:608-616 
21. Chen H, Sullivan G, Yue LQ, Katz A, Quon MJ: QUICKI is a useful index of insulin sensitivity in subjects with hypertension. Am J Physiol 2003, 4: E804-E812.

22. Katz A, Nambi SS, Mather K, Baron AD, Follmann DA, Sullivan G, Quon MJ: Quantitative insulin sensitivity check index: a simple, accurate method for assessing insulin sensitivity in humans. J clin Endocrinol Metab 2000, 85:2402-2410

23. Jessen N, Pold R, Buhl ES, Jensen LS, Schmitz O, Lund S: Effects of AICAR and exercise on insulin-stimulated glucose uptake, signalling, and GLUT4 content in rat muscles. J Appl Physiol 2003, 94:1373-1379.

24. Hou CW, Chou SW, Ho HY, Lee WC, Lin CH, Kuo CH: Interactive effect of exercise training and growth hormone administration on glucose tolerance and muscle GLUT4 protein expression in rats. J Biomed Sci 2003, 10:689-696.

25. Yamaguchi Y, Tanaka S, Yamakawa T, Kimura M, Ukawa K, Yamada Y, Ishihara M, Sekihara H: Reduced serum dehydroepiandrosterone levels in diabetic patients with hyperinsulinaemia. Clin Endocrinol 1998, 49:377-383.

26. Ivy JL, Young JC, Craig BW, Kohrt WM, Holloszy JO: Ageing, exercise and food restriction: effects on skeletal glucose uptake. Mech Ageing Dev 1991, 61:123-133.

27. Sanchez J, Heredia FP, Priego T, Portillo MP, Zamora S, Garaulet M, Palou A. Dehydroepiandrosterone prevents age-associated alterations, increasing insulin sensitivity. J Nutr Biochem 2008, 19:809-818.

28. Jedrzejuk D, Medras M, Milewicz A, Demissie M: Dehydroepiandrosterone replacement in health men with age-related decline of DHEA-S: effects of fat distribution, insulin sensitivity and lipid metabolism. Aging male 2003, 6:151-156.

29. Villareal DT, Hollszy JO: Effect of DHEA on abdominal fat and insulin action in elderly women and men. JAMA 2004, 292:2243-2248.

30. Nair KS, Rizza RA, O'Brien P, Dhatariya K, Short KR, Nehra A, Vittone JL, Klee GG, Basu A, Basu R, Cobelli C, Toffolo G, Dalla Man C, Tindall DJ, Melton LJ 3rd, Smith GE, Khosla S, Jensen MD: DHEA in elderly women and DHEA or testosterone in elderly men. N Engl J Med 2006, 355:1647-1659.

doi:10.1186/1743-7075-9-47

Cite this article as: Sato et al.: DHEA administration and exercise training improves insulin resistance in obese rats. Nutrition \& Metabolism 2012 9:47.

\section{Submit your next manuscript to BioMed Central and take full advantage of:}

- Convenient online submission

- Thorough peer review

- No space constraints or color figure charges

- Immediate publication on acceptance

- Inclusion in PubMed, CAS, Scopus and Google Scholar

- Research which is freely available for redistribution 